\title{
Safety and Efficacy of Repeat Administration of Triamcinolone Acetonide Extended-release in Osteoarthritis of the Knee: A Phase 3b, Open-label Study
}

\author{
Andrew I. Spitzer - John C. Richmond - Virginia B. Kraus • \\ Andreas Gomoll · Deryk G. Jones · Kim M. Huffman • \\ Charles Peterfy $\cdot$ Amy Cinar $\cdot$ Joelle Lufkin $\cdot$ Scott D. Kelley
}

Received: December 22, 2018 / Published online: February 11, 2019

(C) The Author(s) 2019

\section{ABSTRACT}

Introduction: The aim of this work is to assess the safety and efficacy of repeat administration of triamcinolone acetonide extended-release (TA-ER) in patients with symptomatic knee osteoarthritis (OA), including those with advanced radiographic severity.

Methods: In this phase 3b, single-arm, openlabel study, patients aged $\geq 40$ years received the first intra-articular TA-ER injection on day 1. Patients received the second injection timed to the response to the first injection (at either week

Enhanced digital features To view enhanced digital features for this article go to https://doi.org/10.6084/ m9.figshare.7604717.

Electronic supplementary material The online version of this article (https://doi.org/10.1007/s40744019-0140-z) contains supplementary material, which is available to authorized users.

A. I. Spitzer $(\bowtie)$

Cedars-Sinai Orthopedic Center, Los Angeles, CA, USA

e-mail: andrew.spitzer@cshs.org

J. C. Richmond

New England Baptist Hospital, Boston, MA, USA

V. B. Kraus - K. M. Huffman

Duke University School of Medicine, Durham, NC, USA

A. Gomoll

Hospital for Special Surgery, New York, NY, USA
$12,16,20$, or 24). Patients who received two injections were evaluated every 4 weeks for 52 weeks. Safety was evaluated via treatmentemergent adverse events and any change at 52 weeks in index-knee radiographs (chondrolysis, osteonecrosis, insufficiency fractures, subchondral bone changes). Exploratory efficacy endpoints included Western Ontario and McMaster Universities Osteoarthritis Index (WOMAC)-A (pain), -B (stiffness), -C (function), and Knee Injury and Osteoarthritis Outcome Score-Quality of Life (KOOS-QoL) after each injection. Initiative in Methods, Measurements and Pain Assessment in Clinical Trials (IMMPACT) criteria were used to determine moderate and substantial treatment response.

Results: A total of 208 patients were enrolled and received the first injection of TA-ER; 179 $(86.1 \%)$ received the second injection (median time to second injection: 16.6 weeks). Both

\author{
D. G. Jones \\ Ochsner Sports Medicine Institute, New Orleans, \\ LA, USA \\ C. Peterfy \\ Spire Sciences, Inc., Boca Raton, FL, USA \\ A. Cinar · S. D. Kelley \\ Flexion Therapeutics, Inc., Burlington, MA, USA \\ J. Lufkin \\ Independent Consultant, Beverly, MA, USA
}


injections were well tolerated, with no unexpected adverse events or significant radiographic changes at week 52 . The magnitude and duration of clinical benefit after the first and second injections were similar, and most patients reported a substantial $(\geq 50 \%)$ analgesic response after both doses.

Conclusions: Repeat administration of TA-ER using a flexible dosing schedule timed to patient response was well tolerated, with no radiographic evidence of cartilage impact. Both injections resulted in similar improvements in OA symptoms across a broad spectrum of disease severity reflective of that seen in clinical practice.

\section{Trial Registration Information:}

ClinicalTrials.gov identifier: NCT03046446.

Funding: Flexion Therapeutics, Inc.

Plain Language Summary: Plain language summary available for this article.

Keywords: Clinical study; Corticosteroid injection; Knee osteoarthritis; Safety

\section{PLAIN LANGUAGE SUMMARY}

Injecting steroids into knees can improve pain and stiffness for patients with knee osteoarthritis. Unfortunately, the relief may be brief, because the drug does not stay in the joint for very long. As a result, many patients will get another injection when the pain returns. There is a new form of steroid that helps the drug stay in the joint longer by embedding the steroid in a biodegradable bead (TA-ER). The safety of repeat injections of this new product was tested in patients with knee osteoarthritis. All patients were given a knee injection of TA-ER. Patients who experienced pain relief with the first injection could receive the second injection 3-6 months later, depending on when the benefit from the first injection waned. The second injection was not given to patients with benefits from the first injection that lasted longer than 24 weeks or who had no benefit from the first injection. The second injection was given to most patients. For three-quarters of the patients, the time between injections was at least 4 months. Both injections were well tolerated and there was little difference in side effects between the first and second injections. Pain was reduced by more than half for most patients and the amount of pain relief and time it lasted were about the same following both injections. Results from this study will help doctors know when to provide a second injection of TA-ER for osteoarthritis pain in the knee.

\section{INTRODUCTION}

Patients with osteoarthritis (OA) experience joint pain, tenderness, stiffness, and limited movement; pain and functional limitations worsen with disease progression and, in many cases, can ultimately necessitate total joint arthroplasty [1]. The American College of Rheumatology (ACR), the Osteoarthritis Research Society International, and the European League against Rheumatism recommend the use of intra-articular (IA) corticosteroids (CS) for short-term acute pain relief in OA [2-4]. For patients with $\mathrm{OA}$ of the knee, traditional IACS provide local pain relief by targeting the inflamed synovium [5]. However, efflux of IACS from the joint begins within hours of IA injection of standard crystalline formulations $[6,7]$, limiting the potential magnitude and persistence of analgesia and possibly causing systemic effects [6].

In clinical practice, repeat IACS administration is common because of the relatively short duration of symptom relief against the backdrop of the chronic (often decades-long) nature of OA. Repeat injections of traditional IACS have demonstrated an acceptable safety profile in terms of discontinuation rates, postinjection steroid flare, local discomfort, and radiographically assessed joint deterioration [8-11]; however, questions remain regarding the potential for joint infection [8, 9], short-term hyperglycemia in patients with diabetes $[12,13]$, and cartilage loss [14, 15], as well as diminished efficacy in patients with a history of prior IACS use in some studies $[16,17]$.

Triamcinolone acetonide extended-release (TA-ER; formerly FX006) is a novel poly(lacticco-glycolic acid) (PLGA) microsphere-based formulation of TA for IA injection that was recently approved by the US Food and Drug 
Administration to treat pain associated with $\mathrm{OA}$ of the knee [18]. In phase 2 studies, a single TAER IA injection maintained measurable TA synovial fluid concentrations through 12 weeks after injection, whereas after an initial injection of standard TA crystalline suspension (TAcs) synovial TA concentrations were below the limit of quantification by 6 weeks [19]. The prolonged joint residency associated with TAER appears to contribute to its efficacy profile as demonstrated in a large, randomized controlled phase 3 study, where clinically meaningful improvements in pain, stiffness, function, and quality of life were observed through 12 weeks after a single IA injection of TA-ER compared with saline-placebo and traditional TAcs [20]. In addition, pain relief with TA-ER was significantly greater than for saline-placebo through 16 weeks [20]. To date, the TA-ER safety profile appears similar to that observed for saline-placebo and traditional TAcs [20].

The current open-label, single-arm, phase 3b study in patients with symptomatic knee OA (ClinicalTrials.gov identifier: NCT03046446) was designed to assess the safety of repeat administration of TA-ER with 1 year of followup. In contrast with prior studies conducted to evaluate repeat administration of traditional IACS at fixed intervals $[10,15]$, this study used a flexible dosing schedule timed to patient response. In addition, prespecified exploratory efficacy endpoints were included to assess individual patient responses to symptomatic treatment via Western Ontario and McMaster Universities Osteoarthritis Index (WOMAC)-A (pain), -B (stiffness), and -C (function) subscale scores as well as changes in quality of life (QoL).

\section{METHODS}

\section{Design}

This phase 3b, single-arm, open-label study (Fig. 1) was conducted at 17 study centers (including clinical research centers, community clinics, and academic hospitals) in the United States. All procedures were conducted in accordance with the ethical standards of the responsible committee on human experimentation (institutional and national) and with the Helsinki Declaration of 1975 , as revised in 2000 . The protocol was approved by governing ethical bodies at each participating site (see Supplemental Materials), and patients provided written informed consent before participating in any study-related procedures.

\section{Participant Characteristics}

Key inclusion criteria included age $\geq 40$ years, symptomatic knee OA for $\geq 6$ months, an ACR clinical and radiologic diagnosis of OA [21], Kellgren-Lawrence grade 2-4, WOMAC-A total sum score $\geq 6$, and index-knee pain (designated as the one that was most painful by patients with bilateral disease) for $>15$ days during the previous month.

Key exclusion criteria included reactive arthritis, rheumatoid arthritis, psoriatic arthritis, ankylosing spondylitis, or arthritis associated with inflammatory bowel disease, history of infection in the index-knee joint, clinical signs of active knee infection or crystal disease of the index knee within 1 month, presence of surgical hardware or other foreign body in the index knee, an unstable index-knee joint, or history of or active malignancy within the prior 5 years (except for resected basal cell carcinoma, squamous cell carcinoma of the skin, or effectively managed cervical carcinoma). Patients were not eligible if they had previously received index-knee IACS within 3 months or indexknee IA hyaluronic acid within 6 months, intravenous/intramuscular CS within 3 months, or oral CS within 1 month. Patients were also excluded if they had received treatment with any other IA drug or biologic (e.g., platelet-rich plasma, stem cells, prolotherapy, amniotic fluid) within 6 months or five half-lives, whichever was longer; immunomodulators, immunosuppressives, or chemotherapy within 5 years; or live or attenuated vaccines within 3 months.

Patients were not permitted to receive IACS in any joint (other than study drug in the index knee), any other IA intervention (e.g., injection, aspiration that was unrelated to TA-ER 


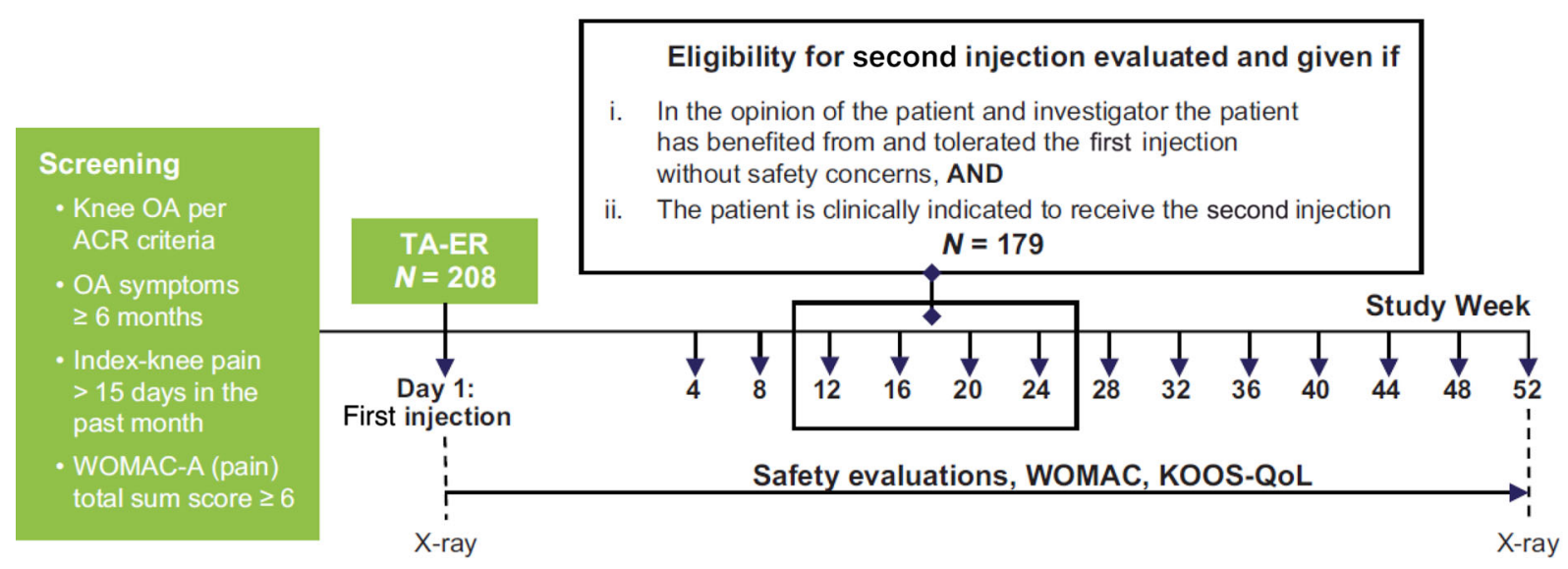

Fig. 1 Study design. ACR American College of Rheumatology, KOOS-QoL Knee injury and Osteoarthritis Outcome Score-Quality of Life, $O A$ osteoarthritis, TA-ER

treatment) in the index knee, or opiates during study participation.

\section{Interventions}

After the first IA injection of TA-ER $32 \mathrm{mg}$ in the index knee (day 1), patients were evaluated every 4 weeks until study completion (see below) or discontinuation. Patients were able to receive the second injection at week $12,16,20$, or 24 depending on when repeat administration criteria were met. Eligibility criteria for the second injection included: (1) benefited from the first injection in the opinion of patient and investigator; (2) no major safety concerns after the first injection as assessed by the investigator; (3) patient was clinically indicated to receive the second injection in the opinion of patient and investigator; and (4) WOMAC-A (pain) total sum score $\geq 6$ or a clinical rationale as to why the second injection was indicated (also see Table S1). Patients who received the second injection were followed for a total of 52 weeks after the first injection, irrespective of when they received a second injection. Those who did not benefit from the first injection completed the study at week 12 . Patients who derived clinical benefit but did not meet repeat administration criteria by week 24 completed the study at week 24 . triamcinolone acetonide extended-release, WOMAC Western Ontario and McMaster Universities Osteoarthritis Index

\section{Procedures}

TA-ER was prepared as a 5-ml injection. TA-ER was administered by the assigned injector, who chose the position of the knee (e.g., extended or flexed), the approach for the injection (e.g., medial or lateral), and the numbing agent for the skin (e.g., topical ethyl chloride, subcutaneous lidocaine) based on standard of care. IA anesthetics were prohibited. Ultrasound guidance was not required. After aspiration of synovial fluid from the index knee was performed or attempted, $5 \mathrm{ml}$ of the reconstituted TA-ER was injected into the synovial space. Patients were asked to avoid strenuous activities or prolonged weight-bearing activities for approximately $24-48 \mathrm{~h}$ after injection and to maintain a stable level of physical activity during study participation.

\section{Study Assessments}

\section{Safety Assessments}

Adverse events (AEs) spontaneously reported by the patient or discovered by the investigator were documented beginning with administration of study drug on day 1 through the last study visit. In addition, baseline and end of study (week 52 or early termination) index-knee radiographs (fixed-flexion posterior anterior 
view, weight-bearing using a standardized kneepositioning device [i.e., KneeFlexor frame] to ensure consistency in patient positioning and image quality) for patients who received two injections were centrally evaluated for joint space narrowing, potential chondrolysis (i.e., rapid progressive joint space narrowing), osteonecrosis, subchondral bone changes, and insufficiency fracture.

\section{Exploratory Efficacy Assessments}

Before all other scheduled assessments, patients completed the WOMAC instrument (Likert 3.1, 5-point scale)-to assess pain (WOMAC-A), stiffness (WOMAC-B), and function (WOMACC) [22] —and the Knee injury and Osteoarthritis Outcome Score-Quality of Life (KOOS-QoL) subscale [23]. The WOMAC and KOOS-QoL questionnaires were administered at screening (WOMAC only) and day 1 and then every 4 weeks through study completion.

\section{Data Analyses}

Analyses were performed for the period from baseline to week 52, the first injection period (from day 1 to the second injection at week 12, week 16 , week 20 , or week 24), the second injection period (from the time of the second injection to a comparable amount of follow-up after the second injection [i.e., 12, 16, 20, or 24 weeks after the second injection]), and from the end of the second injection period to week 52 .

\section{Sample Size}

Based on previous experience in TA-ER clinical trials and assuming that $66 \%$ of patients would receive a second TA-ER injection and that $75 \%$ of those patients would complete the study through week 52 [20], the planned enrollment for this study was 200 patients to ensure that at least 100 patients would receive 2 TA-ER injections and complete the study through week 52 . No formal power and sample size calculations were performed because the efficacy analysis in the current study was considered exploratory and hypothesis generating for potential future studies of repeat administration of TA-ER.

\section{Analysis Populations}

The safety population included all patients who received study drug. The efficacy population included all patients who received study drug and $h a d \geq 1$ post-day 1 efficacy endpoint assessment.

\section{Safety Analyses}

Any treatment-emergent AE (TEAE) with onset after study drug injection or any event that was present at baseline that worsened in intensity through the end of the study was coded using the Medical Dictionary for Regulatory Activities. The proportion of patients reporting TEAEs after the first and second TA-ER injections was determined for patients who received two injections.

Two radiologists independently assessed baseline and end-of-study (week 52 or early termination) index-knee radiographs side by side in random order (time-blinded) for change in joint space narrowing and subchondral bone changes (sclerosis and/or bone attrition) using the Osteoarthritis Research Society International scoring atlas [24]. Joint space narrowing was scored in two locations (medial and lateral tibiofemoral compartments) using a four-point scale from none (0; normal appearance) to severe (3; presence of severe narrowing). Chondrolysis was defined as $\geq 2$-grade increase in joint space narrowing score, corresponding to $\geq 50 \%$ decrease in joint space width between baseline and end of study. Subchondral bone changes were scored as absent or present in each of four locations (medial femur, lateral femur, medial tibia, and lateral tibia). Osteonecrosis and insufficiency fracture were scored as absent or present, based on the experience and expert opinion of the reviewer. In the case of a discrepancy between readers, a third radiologist adjudicated the observation.

\section{Exploratory Efficacy Analyses}

Prespecified exploratory efficacy outcomes included duration of benefit from first injection (time between the first and second injections) and magnitude of benefit derived from the first and second TA-ER injections based on WOMAC-A, -B, and -C and KOOS-QoL scores 
during the period after each injection for patients who received two injections. Leastsquares mean changes were calculated from mixed models, modeling change from baseline for each dose group separately with baseline score and study site as covariates.

Absolute WOMAC and KOOS-QoL scores were summarized for patients who received two injections, using day 1 data as baseline for the first injection and data for the study visit at which the second injection was administered for the second injection.

Treatment responses to initial and repeat administration were also assessed using thresholds defined by the Initiative in Methods, Measurements and Pain Assessment in Clinical Trials (IMMPACT) group for clinically important improvements in treatment outcomes. The proportions of patients meeting IMMPACT criteria for moderate ( $>30 \%$ improvement from day 1) and substantial (>50\% improvement from day 1) response were determined based on WOMAC-A measures at 4 weeks after each injection.

\section{RESULTS}

\section{Demographics and Baseline Disease Characteristics}

Enrolled patients generally had longstanding and extensive disease, with more than twothirds of patients with radiographic OA severity of Kellgren-Lawrence grade 3 or 4 at baseline (Tables 1 and S2). Most patients were women (55.8\%). Mean age was 60.8 years, and mean body mass index was $31.4 \mathrm{~kg} / \mathrm{m}^{2}$. Substantial proportions of patients had previously received IACS in the index knee (51.9\%) and/or had a prior surgery/procedure for knee $\mathrm{OA}$ in the index knee (38.9\%); $17.3 \%$ of patients had received IAHA in the index knee previously. Taken together, these baseline characteristics indicate that the patient population included in this trial reflected a "real-world" population of patients with longstanding, symptomatic moderate-to-advanced knee OA.

\section{Patient Disposition}

Among the 283 patients screened, 208 were enrolled and received the first TA-ER injection (Fig. 2). A total of 59 (28.4\%) patients discontinued the study, three of whom discontinued before week 12, 10 (5.1\%) discontinued before receiving the second injection and 46 (25.7\%) discontinued after the second injection but before week 52 . Of the 46 patients who discontinued after the second injection, 17 discontinued during the second injection period and 29 discontinued between the end of the second injection period and week 52 . The most common reason for study discontinuation among patients who received the second injection was disease progression (25 patients), defined as response to the first and second injections but loss of effect sometime after the response to the second injection. All of the discontinuations classified as disease progression occurred $\geq 12$ weeks after the second injection, and most (17/25) occurred between the end of the second injection period and week 52 . A total of 179 patients $(86.1 \%)$ received the second injection, and six (2.9\%) were not clinically indicated for the second injection at week 24 (Fig. 2).

\section{Safety}

Both TA-ER injections were well tolerated, with similar incidence rates of treatment-emergent AEs (TEAEs) after the first (41.9\%) and second (35.2\%) injections. Most TEAEs that occurred after both injections were unrelated to treatment, nonserious, and grade 1 or grade 2 (Table 2). The patterns of TEAEs were consistent with those reported in previous clinical studies with TA-ER $[18,20,25]$; no unexpected TEAEs were reported. The most frequently reported TEAEs for patients who received two injections of TA-ER were arthralgia (any joint; $10.6 \%$ during the first injection period, $19.0 \%$ during the second injection period) and upper respiratory tract infection $(3.9 \%$ during the first injection period, $2.2 \%$ during the second injection period). Two patients reported arthralgia in the index knee considered related to study 
Table 1 Baseline patient and disease characteristics

\begin{tabular}{|c|c|c|c|}
\hline & $\begin{array}{l}\text { Patients who received } \\
\text { one injection }\end{array}$ & $\begin{array}{l}\text { Patients who received } \\
\text { two injections }\end{array}$ & All patients \\
\hline Safety population & $N=29$ & $N=179$ & $N=208$ \\
\hline Women, $n(\%)$ & $20(69.0)$ & $96(53.6)$ & $116(55.8)$ \\
\hline Age (years), mean (SD) & $59.8(10.48)$ & $60.9(8.48)$ & $60.8(8.81)$ \\
\hline Body mass index $\left(\mathrm{kg} / \mathrm{m}^{2}\right)$, mean $(\mathrm{SD})$ & $31.5(4.72)$ & $31.3(4.89)$ & $31.4(4.86)$ \\
\hline \multicolumn{4}{|l|}{ Type of knee osteoarthritis, $n$ (\%) } \\
\hline Unilateral & $9(31.0)$ & $75(41.9)$ & $84(40.4)$ \\
\hline Bilateral & $20(69.0)$ & $104(58.1)$ & $124(59.6)$ \\
\hline Years since primary diagnosis, mean (SD) & $10.6(9.17)$ & $8.3(7.22)$ & $8.6(7.53)$ \\
\hline Days in past month with index-knee pain, mean (SD) & $27.7(4.90)$ & $28.3(3.76)$ & $28.2(3.93)$ \\
\hline \multicolumn{4}{|l|}{ Index-knee Kellgren-Lawrence grade, $n(\%)$} \\
\hline Grade 2 & $11(37.9)$ & $56(31.3)$ & $67(32.2)$ \\
\hline Grade 3 & $10(34.5)$ & $68(38.0)$ & $78(37.5)$ \\
\hline Grade 4 & $8(27.6)$ & $55(30.7)$ & $63(30.3)$ \\
\hline Prior index-knee surgery/procedure, $n(\%)$ & $7(24.1)$ & $74(41.3)$ & $81(38.9)$ \\
\hline Prior index-knee IACS, $n(\%)$ & $13(44.8)$ & $95(53.1)$ & $108(51.9)$ \\
\hline Prior index-knee IAHA, $n(\%)$ & $4(13.8)$ & $32(17.9)$ & $36(17.3)$ \\
\hline Efficacy population & $N=29$ & $N=178$ & $N=208$ \\
\hline \multicolumn{4}{|l|}{ Outcome measures at baseline, mean (SD) } \\
\hline WOMAC-A (pain) (0-4 Likert) & $2.21(0.572)$ & $2.15(0.580)$ & $2.17(0.586)$ \\
\hline WOMAC-B (stiffness) (0-4 Likert) & $2.33(0.735)$ & $2.48(0.715)$ & $2.48(0.726)$ \\
\hline WOMAC-C (function) (0-4 Likert) & $2.16(0.661)$ & $2.18(0.613)$ & $2.19(0.630)$ \\
\hline KOOS-QoL ${ }^{\mathrm{a}}$ & $24.35(16.565)$ & $28.92(15.872)$ & $27.82(16.057)$ \\
\hline
\end{tabular}

${ }^{a}$ Normalized scores (100 indicating no symptoms and 0 indicating extreme symptoms) were calculated as: 100-AVER$\mathrm{AGE}(\mathrm{Q} 1-\mathrm{Q} 4) / 4 \times 100$

$I A C S$ intra-articular corticosteroids, $I A H A$ intra-articular hyaluronic acid, KOOS-QoL Knee Injury and Osteoarthritis Outcome Score-Quality, $S D$ standard deviation, WOMAC Western Ontario and McMaster Universities Osteoarthritis Index

treatment during the first injection period $(2$ and 22 days after the first injection); three other patients reported arthralgia that led to study discontinuation during the second injection period $(58,80$, and 146 days after the second injection; all were not related to study drug and none required additional treatment).

The incidence of index-knee TEAEs was low and comparable between the first and second injection periods (14.0 and $17.3 \%)$ as well as from the end of the second injection period to 


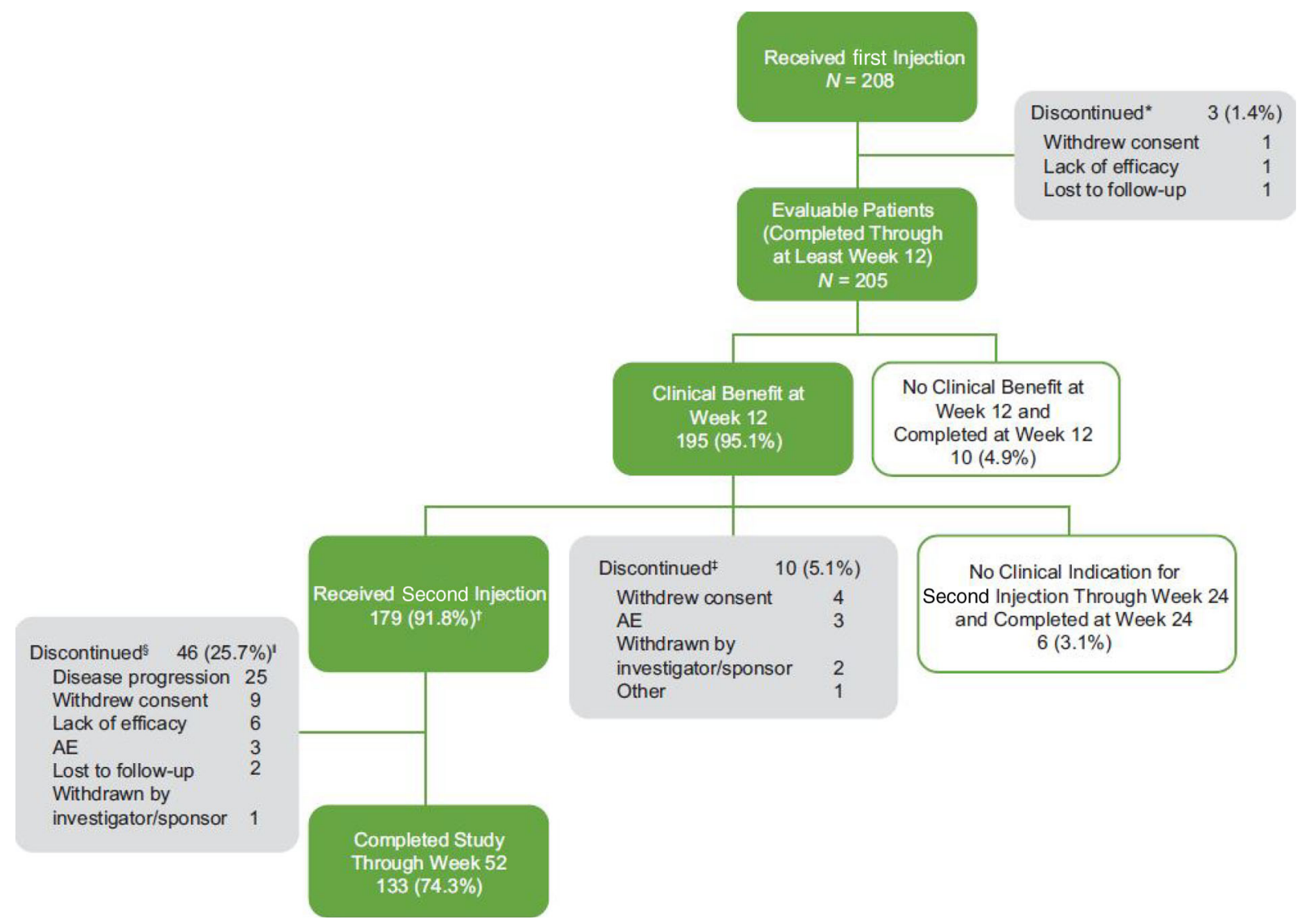

Fig. 2 Patient disposition. ${ }^{*}$ Discontinued before Week 12. †Includes 1 patient who received the second injection after Week 24. IExperienced clinical benefit from the first injection but discontinued before receiving the second injection. $₫$ Received the second injection but discontinued before Week 52 (i.e., 52 weeks after the first injection). I Disease progression defined as response to first and

week 52 (13.4\%) (Table 2). Most index-knee TEAEs were grade 1 or 2 , nonserious and unrelated to study drug; none were consistent with postinjection flare.

Most patients (165/179; 92.2\%) had baseline and end-of-study radiographs (week 52 or at early discontinuation); there were no indications of chondrolysis, osteonecrosis, insufficiency fractures, or clinically significant subchondral bone changes (Table 3).

\section{Exploratory Efficacy}

Of 208 patients who were enrolled and received the first TA-ER injection, $86.1 \%$ received the second injections but loss of effect sometime after the response to the second injection. Lack of efficacy defined as response to the first injection but no response to the second injection. Note: all discontinuations because of disease progression occurred $\geq 12$ weeks after the second injection. $A E$ adverse event

second injection; $2.9 \%$ had persistent pain relief at week 24 such that they had no clinical indication for the second injection, $6.2 \%$ discontinued before receiving the second injection, and $4.8 \%$ had no clinical benefit at week 12 (Fig. 2). The median time to the second injection was 116.5 days ( 16.6 weeks), with a range of 76 to 186 days. The second injection was administered at week 12 for 45 (25.1\%) patients, week 16 for 60 (33.5\%) patients, week 20 for 37 (20.7\%) patients, or week 24 for 36 (20.1\%) patients (Fig. 3).

Among those who received two injections, similarly marked improvements from baseline in WOMAC-A (pain) scores were observed at 4 weeks after both the first and the second injections and then faded during each injection 
Table 2 Summary of adverse events (safety population)

\begin{tabular}{|c|c|c|c|c|}
\hline & \multicolumn{4}{|c|}{ Patients who received two injections $(N=179)$} \\
\hline & $\begin{array}{l}\text { Baseline to } \\
\text { week } 52\end{array}$ & $\begin{array}{l}\text { First injection } \\
\text { period }^{\mathrm{a}}\end{array}$ & $\begin{array}{l}\text { Second injection } \\
\text { period }^{\mathbf{b}}\end{array}$ & $\begin{array}{l}\text { End of second injection } \\
\text { period to week } 52^{c}\end{array}$ \\
\hline Number of TEAEs, $n$ & 336 & 141 & 109 & 86 \\
\hline \multicolumn{5}{|l|}{ Patients with TEAEs, $n$ (\%) } \\
\hline$\geq 1 \mathrm{TEAE}$ & $118(65.9)$ & $75(41.9)$ & $63(35.2)$ & $47(26.3)$ \\
\hline$\geq 1$ serious TEAE & $2(1.1)$ & $1(0.6)$ & $1(0.6)$ & 0 \\
\hline Discontinuations because of TEAE & $3(1.7)$ & 0 & $2(1.1)$ & $1(0.6)$ \\
\hline \multicolumn{5}{|l|}{ TEAEs by maximum severity } \\
\hline Grade 1 & $52(29.1)$ & $43(24.0)$ & $30(16.8)$ & $18(10.1)$ \\
\hline Grade 2 & $58(32.4)$ & $31(17.3)$ & $30(16.8)$ & $25(14.0)$ \\
\hline Grade 3 & $7(3.9)$ & $1(0.6)$ & $2(1.1)$ & $4(2.2)$ \\
\hline Grade 4 & $1(0.6)$ & 0 & $1(0.6)$ & 0 \\
\hline Treatment-related TEAE ${ }^{\mathrm{d}}$ & $10(5.6)$ & $9(5.0)$ & $2(1.1)$ & 0 \\
\hline \multicolumn{5}{|c|}{ Patients with most frequently reported TEAEs ( $\geq 5 \%$ of patients from baseline to week 52 ), $n$ (\%) } \\
\hline Arthralgia (any joint) & $55(30.7)$ & $19(10.6)$ & $34(19.0)$ & $16(8.9)$ \\
\hline Upper respiratory tract infection & $16(8.9)$ & $7(3.9)$ & $4(2.2)$ & $6(3.4)$ \\
\hline \multicolumn{5}{|l|}{ Patients with index-knee TEAEs, $n(\%)$} \\
\hline$\geq 1$ index-knee TEAE & $62(34.6)$ & $25(14.0)$ & $31(17.3)$ & $24(13.4)$ \\
\hline$\geq 1$ serious index-knee TEAE & 0 & 0 & 0 & 0 \\
\hline Discontinuations because of index-knee TEAE & $3(1.7)$ & 0 & $2(1.1)$ & $1(0.6)$ \\
\hline \multicolumn{5}{|l|}{ Index-knee TEAEs by maximum severity } \\
\hline Grade 1 & $24(13.4)$ & $17(9.5)$ & $12(6.7)$ & $6(3.4)$ \\
\hline Grade 2 & $33(18.4)$ & $8(4.5)$ & $18(10.1)$ & $14(7.8)$ \\
\hline Grade 3 & $5(2.8)$ & 0 & $1(0.6)$ & $4(2.2)$ \\
\hline Treatment-related index-knee $\mathrm{TEAE}^{\mathrm{d}}$ & $7(3.9)$ & $7(3.9)$ & $1(0.6)$ & 0 \\
\hline $\begin{array}{l}\text { Patients with } \geq 1 \text { injection procedure-related } \\
\text { TEAE }^{\mathrm{d}}, n(\%)\end{array}$ & $1(0.6)$ & $1(0.6)$ & 0 & 0 \\
\hline
\end{tabular}

Patients were counted once for each period in which they experienced a TEAE (i.e., if a patient experienced a TEAE during both the first and second injection periods, they would be counted for each, but only once for the baseline to week 52 period)

${ }^{a}$ First injection period was from day 1 to week 12 for patients receiving the second injection at week 12 , week 16 for patients receiving the second injection at week 16 , etc

b Second injection period was from the time of the second injection to 12 weeks after the second injection (i.e., week 24) for patients receiving the second injection at week 12, 16 weeks after the second injection (i.e., week 32) for patients receiving the second injection week 16 , etc

${ }^{c}$ End of second injection period to week 52 was from 12 weeks after the second injection (i.e., week 24) to week 52 for patients receiving the second injection at week 12, 16 weeks after the second injection (i.e., week 32) to week 52 for patients receiving the second injection week 16 , etc

${ }^{\mathrm{d}}$ Treatment-related includes TEAEs considered possibly, probably or definitely related to study drug

$T E A E$ treatment-emergent adverse event 
Table 3 Index-knee radiographic analysis (safety population)

\begin{tabular}{|c|c|}
\hline & $\begin{array}{l}\text { Patients } \\
\text { with baseline and end } \\
\text { of study radiographs } \\
(N=165) \\
n(\%)\end{array}$ \\
\hline \multicolumn{2}{|l|}{ Joint space narrowing } \\
\hline 1-point decrease & $1(0.6)$ \\
\hline $1 / 2$-point decrease & $6(3.6)$ \\
\hline No change & $127(77.0)$ \\
\hline $1 / 2$-point increase & $25(15.2)$ \\
\hline 1-point increase & $6(3.6)$ \\
\hline $\begin{array}{l}\geq 2 \text {-point increase } \\
\text { (chondrolysis) }\end{array}$ & 0 \\
\hline \multicolumn{2}{|l|}{ Subchondral bone changes } \\
\hline No change & $163(98.8)$ \\
\hline Absent to absent & $141(85.5)$ \\
\hline Present to present & $22(13.3)$ \\
\hline Absent to present & $1(0.6)^{a}$ \\
\hline Present to absent & $1(0.6)$ \\
\hline \multicolumn{2}{|l|}{ Insufficiency fracture } \\
\hline No change (absent to absent) & $165(100)$ \\
\hline \multicolumn{2}{|l|}{ Osteonecrosis } \\
\hline No change (absent to absent) & $165(100)$ \\
\hline
\end{tabular}

a Considered to be related to progression of osteoarthritis, rather than a treatment-related change because appearance was nonspecific and severe joint space narrowing was observed for both the baseline and end-of-study radiographs

period (Fig. 4 and Table S3). Consistent with decreased pain, marked improvements in stiffness, physical function, and quality of life were observed following both TA-ER injections (Figs. S1-S3 and Table S3). Furthermore, the duration of clinical benefit from the first injection appeared to be similar to the duration of benefit from the second injection for each efficacy endpoint (Figs. 4 and S1-S3).
Using IMMPACT criteria, 89.7 and $84.8 \%$ of patients were considered moderate WOMAC-A responders ( $\geq 30 \%$ improvement) at 4 weeks after the first and second injections, respectively, whereas 76.6 and $67.8 \%$ of patients were considered substantial WOMAC-A responders ( $\geq 50 \%$ improvement). Proportions of moderate and substantial WOMAC-A responders at 4 weeks after each injection were similar regardless of the time of the second injection (Fig. 5). Similar patterns were observed for proportions of patients demonstrating moderate and substantial WOMAC-B, -C, and KOOS-QoL responses (Figs. S4-S6).

\section{DISCUSSION}

Repeat IACS administration prompted by OA symptom recurrence and/or re-emergence of inflammatory signs is common in clinical practice. However, most trials for knee OA have evaluated single administration of traditional IACS with short (12-24 weeks) follow-up periods [5, 20, 25-33]. For trials that followed patients for a longer period (through 2 years of treatment with traditional IACS), the injections were administered at a fixed interval (every 3 months), regardless of the timing of the return of OA symptoms $[10,15]$. The extended-release mechanism of TA-ER is novel, and TAcs and TAER (a long-acting IACS) are pharmacokinetically different agents despite sharing the same active ingredient [19]. As such, results from prior studies of TAcs are not applicable as a benchmark for the safety and efficacy of repeat-administration long-acting IACS. The current study design utilized a flexible dosing interval between TA-ER injections tailored to patient symptoms, rather than a fixed interval. This approach is more reflective of clinical practice and the evolving management of knee OA given the recent availability of a long-acting IACS. The 1-year follow-up period from initial TA-ER administration should be sufficient to identify associated TEAEs and clinically significant joint structural changes through standard radiologic evaluation.

Safety data from our study suggest that patients can receive a second TA-ER injection 


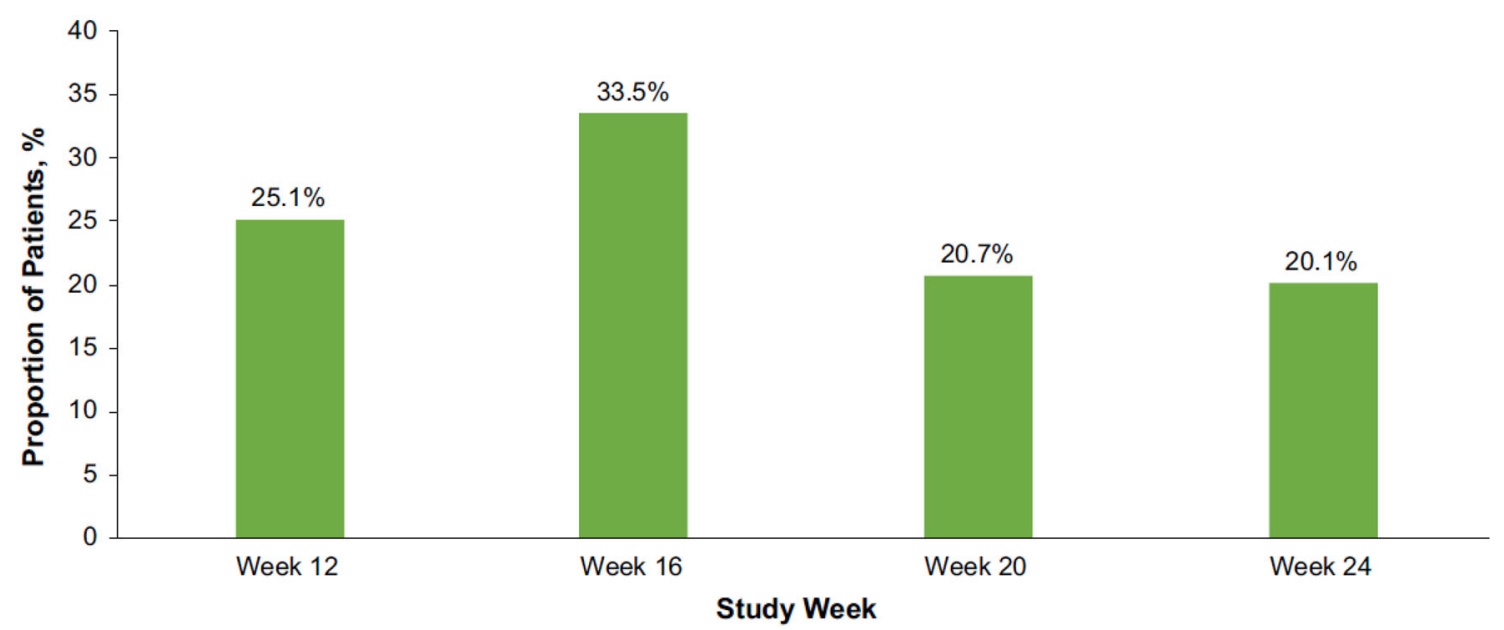

Fig. 3 Timing of the second TA-ER injection for patients who received two injections (efficacy population). Does not include 1 patient who received the second injection after Week 24. TA-ER triamcinolone acetonide extended-release

without experiencing an increase in the incidence of TEAEs. There was no substantial difference in the incidence of TEAEs between the first and second injections, except for slightly more arthralgia events after the second injection, which may indicate the return of OA symptoms as part of the normal pathogenicity of OA. It is notable that patients were not permitted to receive additional TA-ER treatments after the second injection through week 52 . However, the rates of arthralgia TEAEs observed in the current study (10.6\% over $12-24$ weeks after the first injection, $19.0 \%$ over $12-24$ weeks after the second injection) were comparable with what was observed after TA-ER or salineplacebo in the single-administration phase 3 study (14.3 and $12.3 \%$ over 24 weeks, respectively) [20]; only two patients experienced arthralgia that was considered to be related to treatment. Index-knee related AEs were infrequent and indicated no adverse local effect, including no postinjection flares.

Up to 52 weeks of radiographic data collected in this study revealed no adverse effects, including no evidence of osteonecrosis, clinically significant subchondral bone changes, insufficiency fracture, or accelerated joint space narrowing suggestive of chondrolysis. Results from the pivotal phase 3 study showed that the frequency of worsening of joint space narrowing (as assessed semiquantitatively by radiograph) was low and similar among patients receiving TA-ER, TAcs, or saline-placebo [20]. Taken together, the safety data from the current study are reassuring, particularly in light of the prolonged joint residence time associated with TA-ER.

The magnitude of clinical response to both the first and second injections of TA-ER (as assessed via prespecified exploratory endpoints) was similar. This is particularly encouraging given that more than two-thirds of patients who participated in this trial presented with radiographically advanced disease (KellgrenLawrence grade 3 [37.5\%] or grade 4 [30.3\%]). Our results extend those from the phase 3 clinical trial, which enrolled approximately equal proportions of patients with grade 2 $(44.8 \%)$ or grade $3(55.0 \%)$ disease and no patients with grade 4 disease in the TA-ER arm [20]. In addition, patients with higher body mass index and more patients with prior IA treatment/knee procedures were included in the current study than in the pivotal phase 3 trial [20]. As such, participants in the current trial might be more reflective of the types of patients seen in most clinical practices with more advanced and/or prolonged disease. It is interesting to note that, even in this patient population with more advanced disease, the 

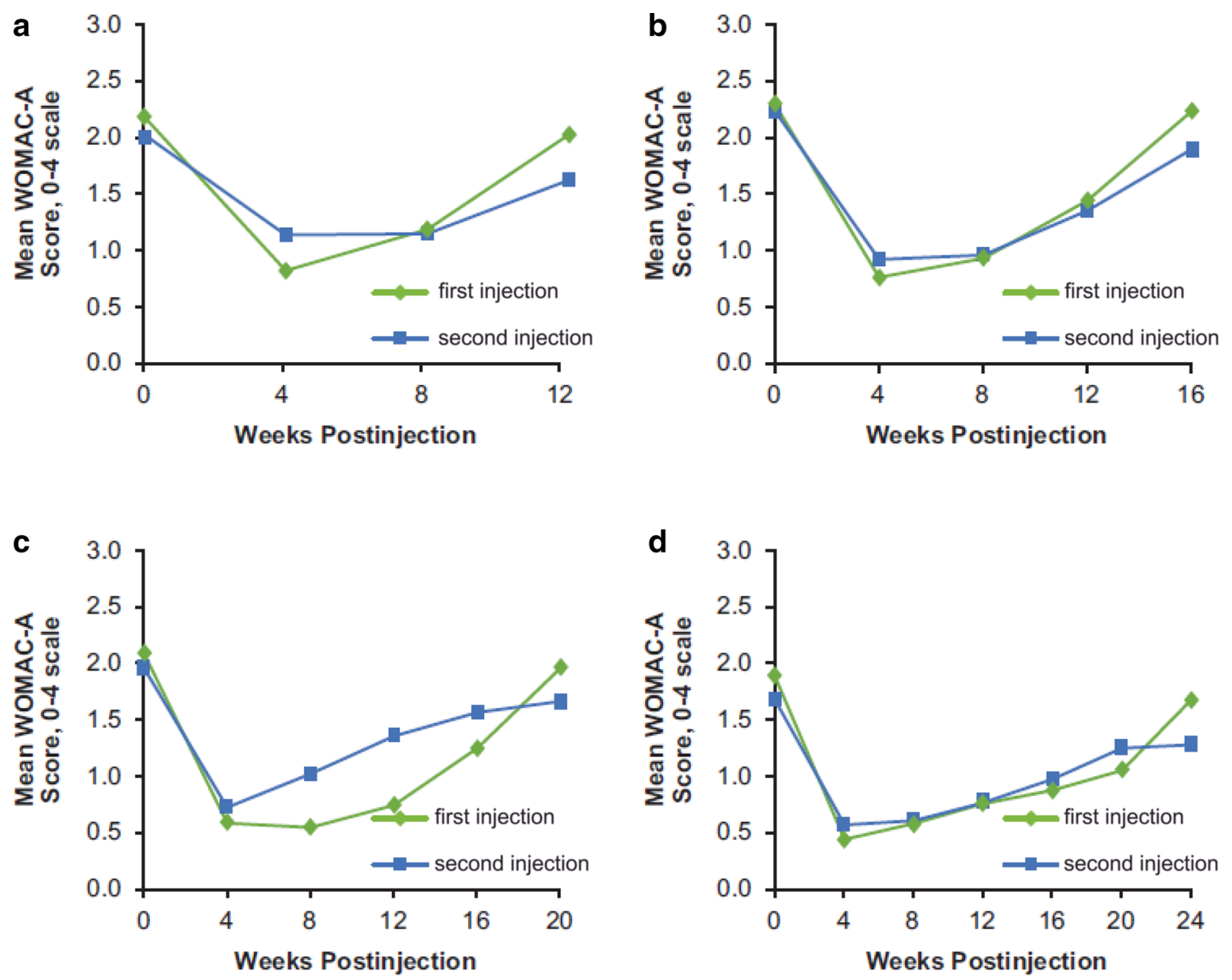

Fig. 4 Comparison of mean WOMAC-A (pain) scores following the first and second TA-ER injections for patients who received the second injection at a week $12(N=45)$, b week $16(N=60)$, c week $20(N=37)$, or d week 24

$(N=36)$ (efficacy population). TA-ER triamcinolone acetonide extended-release, WOMAC Western Ontario and McMaster Universities Osteoarthritis Index

WOMAC and KOOS-QoL responses after the first TA-ER injection are similar to those observed in the phase 3 trial [20]. Furthermore, the clinical benefit achieved after the first TA-ER injection lasted $\geq 16$ weeks for $74 \%$ of patients who received two injections. These data are consistent with the results from the pivotal phase 3 trial, which demonstrated a 16-week duration of benefit from a single TA-ER injection compared with saline-placebo [20].

This study has some limitations. Although the 52-week follow-up period and radiographic assessment provides useful data on the safety

of repeat TA-ER injections, a longer study duration, additional administrations of TA-ER, and magnetic resonance imaging would be useful for assessing certain safety parameters such as changes in the elements of joint structure. In the current study, patients were limited to two injections of TA-ER. Additional administration of TA-ER would have provided additional data and may have improved subject retention. Twenty-five patients discontinued due to pain recurrence sometime after the second injection. All discontinuations due to pain recurrence occurred $>12$ weeks after the second injection, indicating that those patients 
- Moderate Response ( $\geq 30 \%$ Improvement) at 4 Weeks After Injection

- Substantial Response ( $\geq 50 \%$ Improvement) at 4 Weeks After Injection

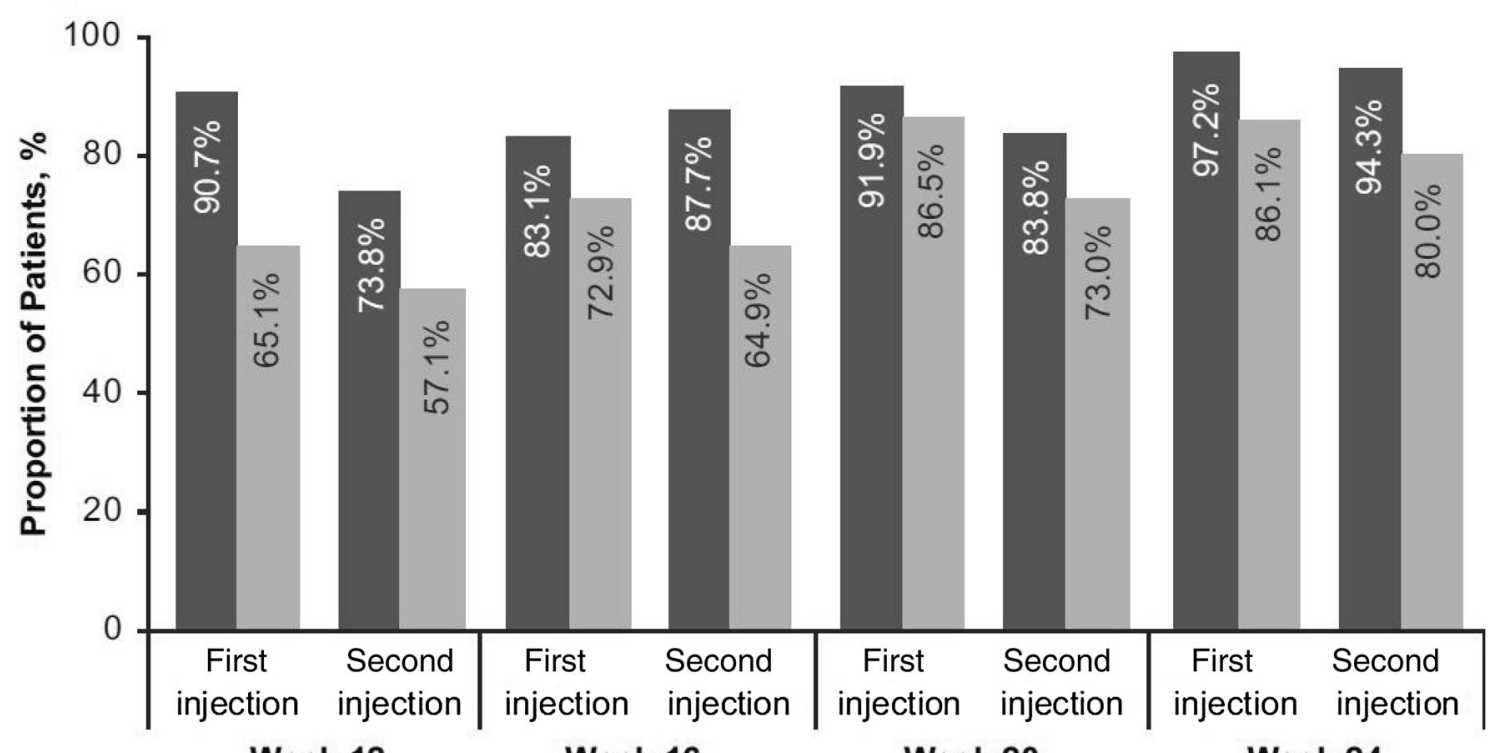

Week 12

Week 16

Week 20

Week 24

Time of the $\mathbf{2}^{\text {nd }}$ Injection

Fig. 5 Proportions of patients achieving moderate ( $\geq 30 \%$ improvement) and substantial ( $\geq 50 \%$ improvement) WOMAC-A (pain) response at 4 weeks after each injection for patients who received the second injection

had responded to the second injection. Permitting a third administration of TA-ER may have reduced the number of discontinuations. Further, as with any open-label, single-arm study, there is potential for bias; however, this was mitigated with respect to the radiographic data by time-blinding the independent radiologists. While inclusion of a placebo arm would have been optimal, the high discontinuation rate expected for patients randomized to placebo who would have been prohibited from receiving other treatment for their knee OA for 1 year (including IACS in any joint, any other IA intervention in the index knee, and opiates) precluded this. Despite these limitations, this study allows for assessment of exploratory efficacy and safety of TA-ER repeat administration under circumstances more closely approximating "real-world" conditions than previous clinical trials. week $12(N=45)$, week $16(N=60)$, week $20(N=37)$, or week $24(N=36)$ (efficacy population). WOMAC Western Ontario and McMaster Universities Osteoarthritis Index

\section{CONCLUSIONS}

In summary, repeat administration of TA-ER using a flexible repeat-dosing schedule tailored to patient response was well tolerated with no unexpected AEs, postinjection flares, or radiographic evidence of deleterious effects on cartilage or other elements of joint structure. In addition, these results indicate that the magnitude and duration of symptom relief after both first and second injections were consistent and similar to those observed in the TA-ER pivotal phase 3 trial. These data demonstrate that individualized care with repeat administration of TA-ER offers patients and physicians a nonopioid option for achieving clinically meaningful control of knee OA symptoms. The treatment strategy utilized in this study can inform clinical decision-making with regard to timing of repeat administration of TA-ER for knee OA pain. 


\section{ACKNOWLEDGEMENTS}

The authors thank the participants in the study.

Funding. This study and all article processing charges were funded by Flexion Therapeutics, Inc., Burlington, MA, USA. All authors had full access to all data in this study and take complete responsibility for the integrity of the data and accuracy of the data analysis.

Medical Writing and/or Editorial Assistance. Professional medical writing and editing assistance was provided by Jennifer Jaworski, MS, (ApotheCom, Yardley, PA, USA); this support was funded by Flexion Therapeutics, Inc. (Burlington, MA, USA). The authors thank Teresa Curto and Ashish Aggarwal (Cytel, Cambridge, MA, USA) as well as Katherine Kacena, PhD (Biobridges, Wellesley, MA, USA) for biostatistical support.

Authorship. All named authors meet the International Committee of Medical Journal Editors (ICMJE) criteria for authorship for this article, take responsibility for the integrity of the work as a whole, and have given their approval for this version to be published.

Disclosures. Andrew I. Spitzer has received research support from DePuy and Flexion Therapeutics, Inc., and consulting fees from DePuy, Flexion Therapeutics, Inc., Sanofi Biosurgical, and Zimmer Biomet. John C. Richmond has received consulting fees from Flexion Therapeutics, Inc. Virginia B. Kraus has received consulting fees from Flexion Therapeutics, Inc. Deryk G. Jones has received research support from Sanofi-Aventis (paid to institution); has received consulting/speaking fees from Genzyme, Mitek, Musculoskeletal Transplant Foundation, and Zimmer Biomet; and is a Musculoskeletal Transplant Foundation board/committee member. Andreas Gomoll has received research support from Joint Restoration Foundation (JRF) and Organogenesis; and consulting fees from JRF, Organogenesis, Vericel, and Flexion Therapeutics, Inc. Charles Peterfy is the owner of Spire Sciences, Inc., a core laboratory providing central image analysis services to multiple pharmaceutical, biotechnology and medical devices companies. Amy Cinar is an employee of Flexion Therapeutics, Inc. and owns stock/stock options in Flexion. Joelle Lufkin is a former employee of Flexion Therapeutics, Inc. and owns stock/stock options in Flexion. Scott D. Kelley is an employee of Flexion Therapeutics, Inc. and own stock/stock options in Flexion. Kim M. Huffman has nothing to disclose.

Compliance with Ethics Guidelines. All procedures were conducted in accordance with the ethical standards of the responsible committee on human experimentation (institutional and national) and with the Helsinki Declaration of 1975 , as revised in 2000. The protocol was approved by governing ethical bodies at each participating site (see Supplemental Materials), and patients provided written informed consent before any study-related procedures.

Data Availability. The datasets generated and/or analyzed within this publication are available from the corresponding author on reasonable request.

Open Access. This article is distributed under the terms of the Creative Commons Attribution-NonCommercial 4.0 International License (http://creativecommons.org/licenses/ by-nc/4.0/), which permits any noncommercial use, distribution, and reproduction in any medium, provided you give appropriate credit to the original author(s) and the source, provide a link to the Creative Commons license, and indicate if changes were made.

\section{REFERENCES}

1. Robinson WH, Lepus CM, Wang Q, et al. Low-grade inflammation as a key mediator of the pathogenesis of osteoarthritis. Nat Rev Rheumatol. 2016;12(10):580-92.

2. Hochberg MC, Altman RD, April KT, et al. American College of Rheumatology. American College of Rheumatology 2012 recommendations for the use of nonpharmacologic and pharmacologic therapies 
in osteoarthritis of the hand, hip, and knee. Arthritis Care Res (Hoboken). 2012;64(4):465-74.

3. Jordan KM, Arden NK, Doherty M, et al. Standing Committee for International Clinical Studies Including Therapeutic Trials ESCISIT. EULAR recommendations 2003: an evidence based approach to the management of knee osteoarthritis: report of a Task Force of the Standing Committee for International Clinical Studies Including Therapeutic Trials (ESCISIT). Ann Rheum Dis. 2003;62(12):1145-55.

4. McAlindon TE, Bannuru RR, Sullivan MC, et al. OARSI guidelines for the non-surgical management of knee osteoarthritis. Osteoarthr Cartil. 2014;22(3):363-88.

5. Juni P, Hari R, Rutjes AW, et al. Intra-articular corticosteroid for knee osteoarthritis. Cochrane Database Syst Rev. 2015;10:5328.

6. Habib GS. Systemic effects of intra-articular corticosteroids. Clin Rheumatol. 2009;28(7):749-56.

7. Derendorf H, Mollmann H, Gruner A, Haack D, Gyselby G. Pharmacokinetics and pharmacodynamics of glucocorticoid suspensions after intraarticular administration. Clin Pharmacol Ther. 1986;39(3):313-7.

8. Seror P, Pluvinage P, d'Andre FL, Benamou P, Attuil G. Frequency of sepsis after local corticosteroid injection (an inquiry on 1160000 injections in rheumatological private practice in France). Rheumatology (Oxford). 1999;38(12):1272-4.

9. Bellamy N, Campbell J, Robinson V, Gee T, Bourne $\mathrm{R}$, Wells G. Intraarticular corticosteroid for treatment of osteoarthritis of the knee. Cochrane Database Syst Rev. 2006(2):CD005328.

10. Raynauld JP, Buckland-Wright C, Ward R, et al. Safety and efficacy of long-term intraarticular steroid injections in osteoarthritis of the knee: a randomized, double-blind, placebo-controlled trial. Arthritis Rheum. 2003;48(2):370-7.

11. Balch HW, Gibson JM, El-Ghobarey AF, Bain LS, Lynch MP. Repeated corticosteroid injections into knee joints. Rheumatol Rehabil 1977;16(3):137-40.

12. Habib G, Safia A. The effect of intra-articular injection of betamethasone acetate/betamethasone sodium phosphate on blood glucose levels in controlled diabetic patients with symptomatic osteoarthritis of the knee. Clin Rheumatol 2009;28(1):85-7.

13. Habib GS, Bashir M, Jabbour A. Increased blood glucose levels following intra-articular injection of methylprednisolone acetate in patients with controlled diabetes and symptomatic osteoarthritis of the knee. Ann Rheum Dis 2008;67(12):1790-1.

14. da Costa BR, Hari R, Juni P. Intra-articular corticosteroids for osteoarthritis of the knee. JAMA 2016;316(24):2671-2.

15. McAlindon TE, LaValley MP, Harvey WF, et al. Effect of intra-articular triamcinolone vs saline on knee cartilage volume and pain in patients with knee osteoarthritis: a randomized clinical trial. JAMA. 2017;317(19):1967-75.

16. Arden NK, Reading IC, Jordan KM, Thomas L, Platten $\mathrm{H}$, Hassan A, et al. A randomised controlled trial of tidal irrigation vs corticosteroid injection in knee osteoarthritis: the KIVIS Study. Osteoarthritis Cartilage 2008;16(6):733-9.

17. McCabe PS, Maricar N, Parkes MJ, Felson DT, O'Neill TW. The efficacy of intra-articular steroids in hip osteoarthritis: a systematic review. Osteoarthritis Cartilage 2016;24(9):1509-17.

18. Zilretta [prescribing information]. Burlington, MA: Flexion Therapeutics, Inc.; 2017.

19. Kraus VB, Conaghan PG, Aazami HA, et al. Synovial and systemic pharmacokinetics (PK) of triamcinolone acetonide (TA) following intra-articular (IA) injection of an extended-release microsphere-based formulation (FX006) or standard crystalline suspension in patients with knee osteoarthritis (OA). Osteoarthr Cartil. 2018;26(1):34-42.

20. Conaghan PG, Hunter DJ, Cohen SB, et al. FX0062014-008 Participating Investigators. Effects of a single intra-articular injection of a microsphere formulation of triamcinolone acetonide on knee osteoarthritis pain: a double-blinded, randomized, placebo-controlled, multinational study. J Bone Joint Surg Am. 2018;100(8):666-77.

21. Altman R, Asch E, Bloch D, et al. Development of criteria for the classification and reporting of osteoarthritis. Classification of osteoarthritis of the knee. Diagnostic and Therapeutic Criteria Committee of the American Rheumatism Association. Arthritis Rheum. 1986;29(8):1039-49.

22. Bellamy N, Buchanan WW, Goldsmith CH, Campbell J, Stitt LW. Validation study of WOMAC: a health status instrument for measuring clinically important patient relevant outcomes to antirheumatic drug therapy in patients with osteoarthritis of the hip or knee. J Rheumatol. 1988;15(12):1833-40.

23. Roos EM, Roos HP, Lohmander LS, Ekdahl C, Beynnon BD. Knee Injury and Osteoarthritis Outcome Score (KOOS)-development of a self-administered outcome measure. J Orthop Sports Phys Ther. 1998;28(2):88-96. 
24. Altman RD, Gold GE. Atlas of individual radiographic features in osteoarthritis, revised. Osteoarthr Cartil. 2007;15:A1-56.

25. Conaghan PG, Cohen SB, Berenbaum F, Lufkin J, Johnson JR, Bodick N. Brief report: a phase IIb trial of a novel extended-release microsphere formulation of triamcinolone acetonide for intraarticular injection in knee osteoarthritis. Arthritis Rheumatol. $2018 ; 70(2): 204-11$.

26. Bodick N, Lufkin J, Willwerth C, et al. An intraarticular, extended-release formulation of triamcinolone acetonide prolongs and amplifies analgesic effect in patients with osteoarthritis of the knee: a randomized clinical trial. J Bone Joint Surg Am. 2015;97(11):877-88.

27. Beyaz SG. Comparison of efficacy of intra-articular morphine and steroid in patients with knee osteoarthritis. J Anaesthesiol Clin Pharmacol. 2012;28(4):496-500.

28. de Campos GC, Rezende MU, Pailo AF, Frucchi R, Camargo OP. Adding triamcinolone improves viscosupplementation: a randomized clinical trial. Clin Orthop Relat Res. 2013;471(2):613-20.

29. Chao J, Wu C, Sun B, et al. Inflammatory characteristics on ultrasound predict poorer longterm response to intraarticular corticosteroid injections in knee osteoarthritis. J Rheumatol. 2010;37(3): $650-5$.

30. Di Sante L, Paoloni M, Dimaggio M, et al. Ultrasound-guided aspiration and corticosteroid injection compared to horizontal therapy for treatment of knee osteoarthritis complicated with Baker's cyst: a randomized, controlled trial. Eur J Phys Rehabil Med. 2012;48(4):561-7.

31. Petrella RJ, Emans PJ, Alleyne J, Dellaert F, Gill DP, Maroney M. Safety and performance of Hydros and Hydros-TA for knee osteoarthritis: a prospective, multicenter, randomized, double-blind feasibility trial. BMC Musculoskelet Disord. 2015;16:57.

32. Yavuz U, Sokucu S, Albayrak A, Ozturk K. Efficacy comparisons of the intraarticular steroidal agents in the patients with knee osteoarthritis. Rheumatol Int. 2012;32(11):3391-6.

33. Henriksen M, Christensen R, Klokker L, et al. Evaluation of the benefit of corticosteroid injection before exercise therapy in patients with osteoarthritis of the knee: a randomized clinical trial. JAMA Intern Med. 2015;175(6):923-30. 